УДК 533.9.082

ЭВОЛЮЦИЯ ЭЛЕКТРИЧЕСКОГО ПОТЕНЦИАЛА И ГЕОДЕЗИЧЕСКИХ АКУСТИЧЕСКИХ МОД В ОМИЧЕСКИХ И ЭЦР-РЕЖИМАХ С ИМПУЛЬСНЫМ НАПУСКОМ ПРИМЕСЕЙ В ТОКАМАКЕ Т-10

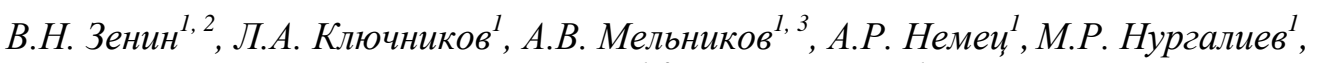
Г.Ф. Субботин ${ }^{1,2}$, Д.А. Шелухин ${ }^{1}$

${ }^{1}$ НИЦ «Курчатовский институт», Москва, Россия

${ }^{2}$ Московский физико-технический институт, Долгопрудный, Россия

${ }^{3}$ Национальный исследовательский ядерный университет «МИФИ», Москва, Россия

Качественно сравниваются геодезические акустические моды (ГАМ) и эволюция среднего уровня электрического потенциала плазмы в токамаке Т-10 в режимах с импульсным (5 мс) напуском различных примесей (гелия, азота и неона). Показано, что напуск примеси меняет уровень потенциала. Также показано, что напуск примеси сильно влияет на ГАМ, резко снижая её амплитуду и снижая частоту. Это влияние продолжается в течение примерно 50 мс, затем амплитуда и частота ГАМ выходят на новый стационарный уровень. Приведены сравнения параметров ГАМ с основными плазменными параметрами и уровнем флуктуаций электронной плотности. Рассмотрена эволюция частоты ГАМ в сравнении с теоретически ожидаемой зависимостью для частоты локальных ГАМ с локальными значениями температуры на периферии.

Ключевые слова: токамак, Т-10, ГАМ, зондирование плазмы пучком тяжёлых ионов.

\title{
PLASMA ELECTRIC POTENTIAL AND GEODESIC ACOUSTIC MODE EVOLU- TION IN OHMIC AND ECRH REGIMES WITH PULSED IMPURITY PUFFING ON THE T-10 TOKAMAK
}

\author{
V.N. Zenin ${ }^{1,2}$, L.A. Klyuchnikov ${ }^{1}$, A.V. Melnikov ${ }^{1,3}$, A.R. Nemets ${ }^{1}$, M.R. Nurgaliev ${ }^{l}$, \\ G.F. Subbotin ${ }^{l, 2}$, D.A. Shelukhin ${ }^{l}$
}

\footnotetext{
${ }^{I}$ NRC «Kurchatov Institute», Moscow, Russia

${ }^{2}$ Moscow Institute of Physics and Technology, Dolgoprudny, Russia

${ }^{3}$ National Research Nuclear University «MEPhI», Moscow, Russia
}

The geodesic acoustic mode (GAM) and the evolution of the average level of the electrostatic plasma potential in the T-10 tokamak in the regime with short $(5 \mathrm{~ms})$ impurity puffing are considered in this work. Plasma potential and GAM evolution are compared qualitatively at helium, nitrogen and neon impurities puffing. It was shown that impurity puffing changes the average plasma potential level. It was also shown that impurity puffing strongly affects GAM properties, reducing its amplitude sharply and decreasing its frequency. This effect lasts for about $50 \mathrm{~ms}$, and then the amplitude and frequency of GAM reaches a new stationary level. The GAM parameters are compared with the evolution of the basic plasma parameters and the turbulence level of electron density. The GAM frequency evolution was considered versus theoretical dependence for local GAM taking into account peripheral temperatures.

Key words: tokamak, T-10, GAM, HIBP.

DOI: $10.21517 / 0202-3822-2016-39-3-91-98$

\section{ВВЕДЕНИЕ}

Актуальным вопросом современного управляемого термоядерного синтеза являются физические механизмы турбулентного транспорта энергии в замкнутых магнитных ловушках. Зональные потоки рассматриваются как возможный механизм саморегуляции турбулентности, влияющий на потери энергии из плазмы [1]. В то же время среднее значение потенциала связано с удержанием плазмы $[2,3]$.

В данной работе рассматриваются геодезические акустические моды (ГАМ), высокочастотная ветвь зональных потоков, которые трансформируют радиальные потери энергии в крутильные колебания плазмы, препятствуя потерям энергии поперёк магнитного поля. ГАМ, в основном, проявляют себя как колебания электростатического потенциала и плотности, при этом амплитуда колебаний потенциала, нормированная на электронную температуру, значительно выше амплитуды колебаний относительной плотности $[4,5]$. Ранее было показано, что параметры ГАМ определяются параметрами плазмы на периферии [6]. Поэтому в данной работе для анализа используются именно краевые значения электронной и ионной температуры и электронной плотности.

Параллельно этому изучается эволюция среднего уровня электростатического потенциала плазмы. 


\section{СХЕМА ЭКСПЕРИМЕНТА}

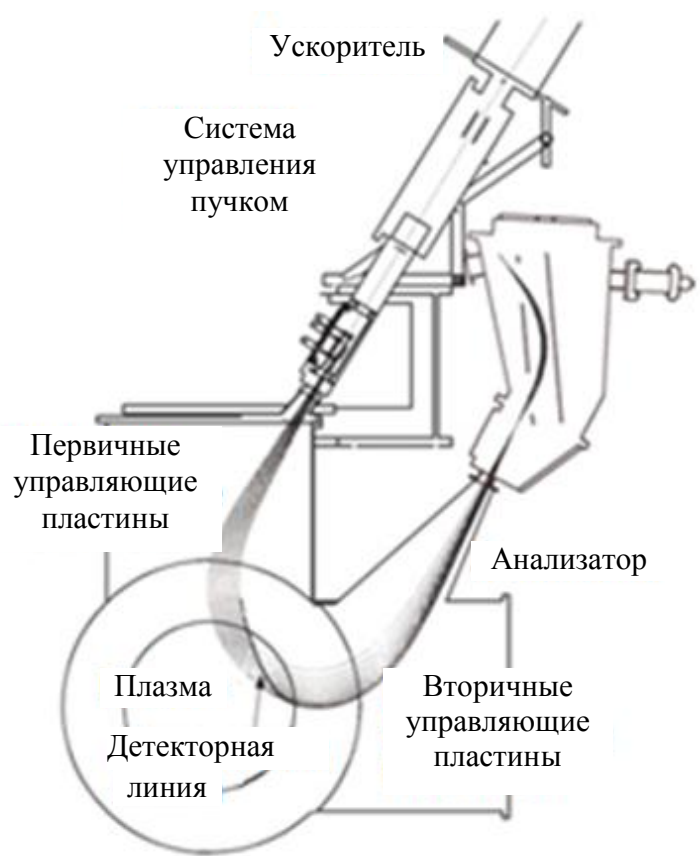

Рис. 1. Схема диагностики НIBР

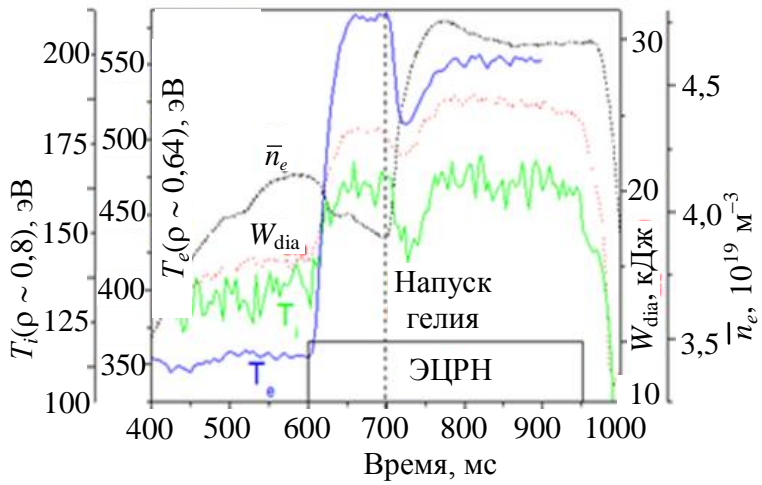

Рис. 2. Эволюция основных параметров плазмы в импульсе № 65489 с напуском Не, энергосодержание измерялось по диамагнитному сигналу $W_{\mathrm{dia}}$
Т-10 является круглым токамаком $(R=1,5 \mathrm{M}, a=0,3 \mathrm{м}$, $B_{0} \sim 2,4$ Тл) с рельсовым углеродным лимитером. Одним из проявлений ГАМ являются колебания радиального электрического поля и, следовательно, потенциала плазмы. Эти колебания в горячей плазме были исследованы с помощью диагностики зондирования пучком тяжёлых ионов (НІВР), уникального метода прямого измерения электрического потенциала (зондирование ионами $\mathrm{Tl}^{+}$ энергией до 300 кэВ). Основные принципы диагностики HIBP описаны в работе [7]. Схема диагностики показана на рис. 1.

Эволюция ГАМ и потенциала представлена на примере импульса № 65489 с плотностью, близкой к предельной $\left(\bar{n}_{e}=(3,5-4,5) 10^{19} \mathrm{M}^{-3}\right)$. Основные плазменные параметры для этого разряда показаны на рис. 2, ток разряда 200 кА. Здесь и далее по тексту $T_{e}$ - электронная температура, $T_{i}-$ ионная температура, $\bar{n}_{e}$ - электронная плотность, $\rho-$ относительный малый радиус. Электронный циклотронный резонансный нагрев (ЭЦРН) длился с 600 по 950 мс, его мощность составляла 1,1 MBт (омическая мощность около 280 кВт). При включении ЭЦРН вырастают электронная и периферийная ионная температуры, а также энергосодержание в плазме. При этом падает среднехордовая плотность, измеренная вдоль центральной хорды, в соответствии с известным эффектом откачки плотности (density pump-out) [8]. В рассматриваемых режимах во время работы гиротрона на 700 мс начинается кратковременный напуск гелия. Напуск продолжался около $3-5$ мс, плотность гелия составляла около $20 \%$ от электронной среднехордовой плотности, измеренной вдоль центральной хорды. Уровень подачи дейтерия при этом снижался и оставался на новом низком уровне до конца импульса. После импульса гелия средняя плотность плазмы вырастала, а значения электронной и ионной температуры снижалось. Энергосодержание в плазме также падало. При этом уровень МГД-активности (по данным зондов и рентгеновского излучения) падает примерно на 25 мс, затем восстанавливается. Пилообразные колебания не испытывают заметного влияния напуска гелия. Уровни температуры и энергосодержания достигают минимума примерно за 20-25 мс, затем восстанавливались до некоторых новых значений за 50-60 мс.
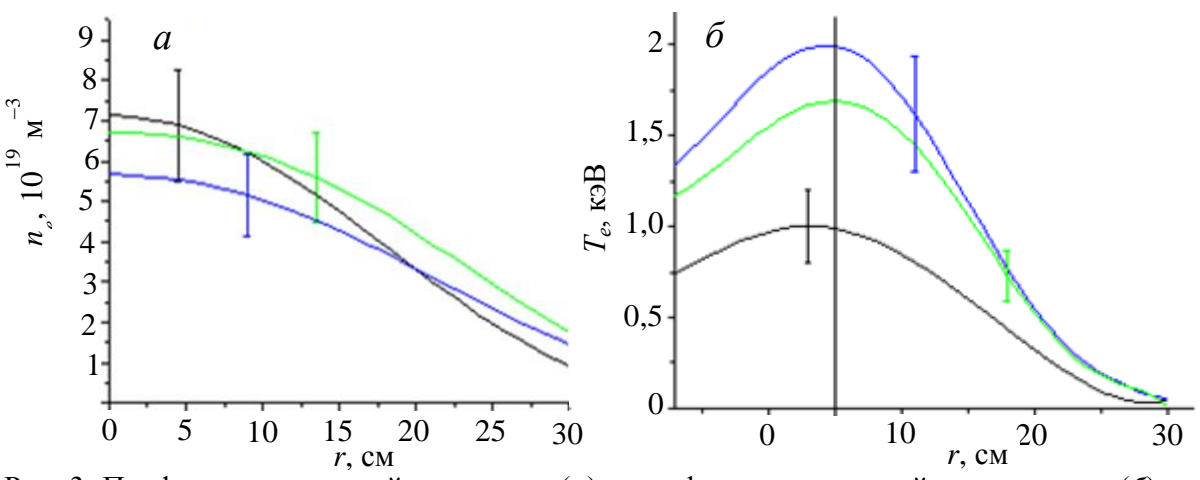

Рис. 3. Профили электронной плотности $(a)$ и профили электронной температуры (б) для различных моментов времени: - — 590 нм до включения ЭЦРН; - -690 нм до напуска гелия; - _ 800 мс после напуска на новом стационаре в импульсе № 65489
На рис. 3, $а$ показаны профили электронной плотности, на рис. 3, 6 - температуры. Виден эффект перестроения профиля плотности после включения гиротрона. После импульсного газонапуска плотность выше на новом стационаре. Включение ЭЦРН увеличивает температуру, после импульсного газонапуска её уровень в центре падает. 


\section{НАБЛЮДЕНИЕ И СВОЙСТВА ГАМ}
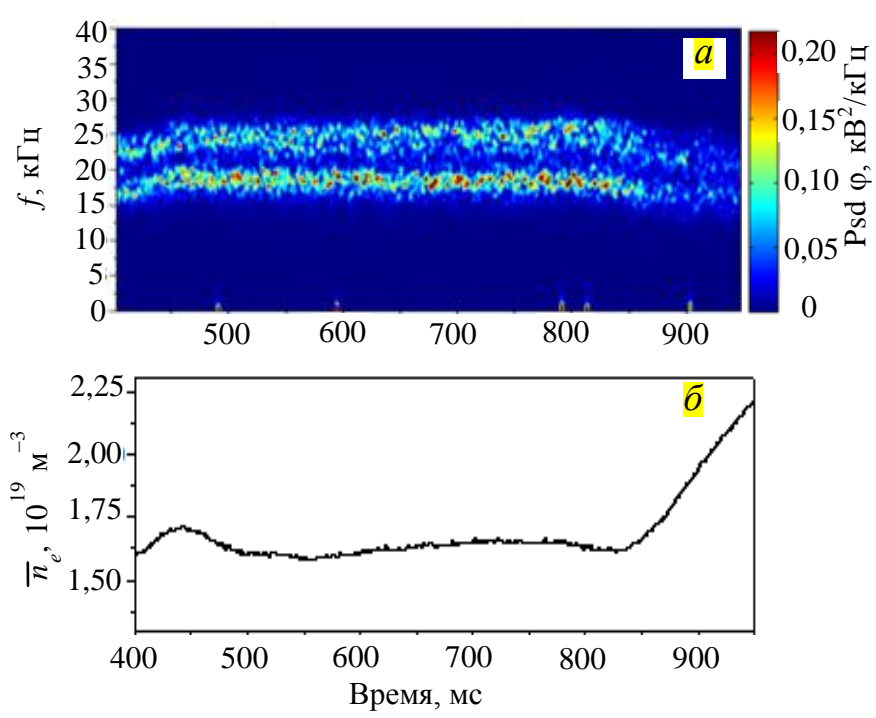

Рис. 4. Спектрограмма плотности мощности колебаний потенциала (a) и среднехордовая плотность плазмы в импульсе № 62759 (б)
Удобным методом представления колебаний являются спектрограммы плотности мощности СПМ (power spectral density (psd)) колебаний. На спектрограммах временная эволюция мощности колебаний в исследуемом частотном диапазоне показана с помощью цветовой шкалы. На рис. 4 показана спектрограмма колебаний потенциала плазмы для импульса № 62759 ( $B=2,4$ Тл, $I_{\mathrm{pl}}=220$ кА, импульс без напуска примесей) на фоне эволюции среднехордовой плотности. Этот импульс представляет собой разряд только с омическим нагревом, на его примере можно увидеть основные черты ГАМ (пик на частоте около 17 кГц). В частности, ГАМ иногда сопровождается несколько более высокочастотным сателлитом. ГАМ и сателлит при низкой плотности представляют собой два контрастных пика на спектрограмме. При увеличении плотности к концу импульса амплитуда ГАМ падает [9]. Увеличение плотности вызывает падение температуры, что, в свою очередь, приводит к падению частоты ГАМ.

На примере этого же импульса проиллюстрировано значение используемых далее понятий амплитуды и частоты ГАМ. Ранее было показано, что ГАМ имеет перемежаемую структуру $[10,11]$ и состоит из стохастической последовательности отдельных вспышек. Поэтому её мгновенная амплитуда сильно меняется за время порядка 1 мс. Кроме того, ГАМ не является монохроматическим пиком (см. рис. 4). Гистограммы распределений амплитуды и частоты ГАМ показаны на рис. 5. На рисунке видно, что распределения имеют гауссовский характер с явно выделенным средним значением и конечной шириной. Для дальнейшего анализа используются усреднённые значения амплитуды и частоты ГАМ.
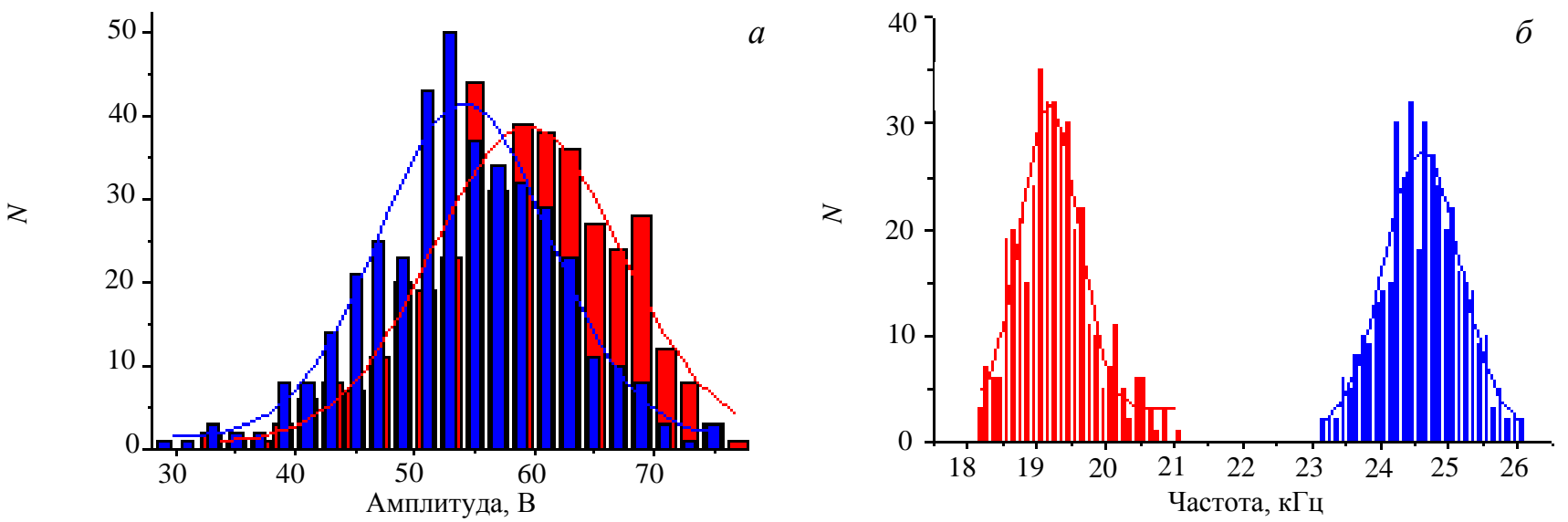

Рис. 5. Распределение мгновенных (разрешение $\sim 1$ мс) амплитуд (a) и частот ГАМ (показана красным цветом) и её сателлита (синим цветом) (б) для импульса № 62759 . Распределения аппроксимированы гауссовыми кривыми: $A_{\mathrm{c}}($ ГАМ) $=59 \mathrm{~B} ; \sigma=8 \mathrm{~B}$; $A_{\mathrm{c}}($ сателлит $)=54 \mathrm{~B} ; \sigma=7$ В; $f_{\mathrm{c}}(Г А М)=19$ кГц; $\sigma=0,43$ кГц; $f_{\mathrm{c}}$ (сателлит) $=24,5$ кГц; $\sigma=0,55$ кГц; $N$ - количество отсчётов. 3десь $A_{\mathrm{c}}$, $f_{\mathrm{c}}$ - среднее значение, $\sigma$ - стандартное отклонение

\section{ЭКСПЕРИМЕНТАЛЬНЫЕ РЕЗУЛЬТАТЫ}

В исследованном режиме средний уровень потенциала и его колебания в частотном диапазоне ГАМ изучались с помощью НІВР. Измерения в импульсе № 65489 проводились на радиусе $r=26$ см. Данные об ионной температуре получены с помощью активной спектроскопической диагностики (CXRS) [12] и пассивной спектроскопии на периферии плазмы [13]. Измерения относительного профиля электронной температуры проводились с помощью ЕСЕ-диагностики. Затем проводилась калибровка данных 


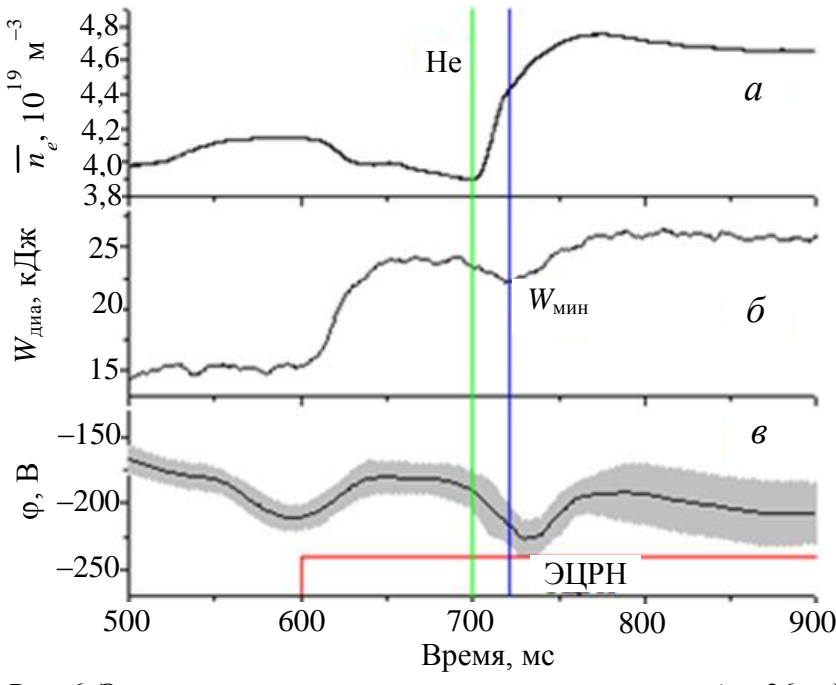

Рис. 6. Эволюция среднего уровня потенциала плазмы $(r=26 \mathrm{~cm})$ (в) в сравнении с эволюцией электронной среднехордовой плотности $(a)$ и эволюцией содержания энергии в плазме (б)
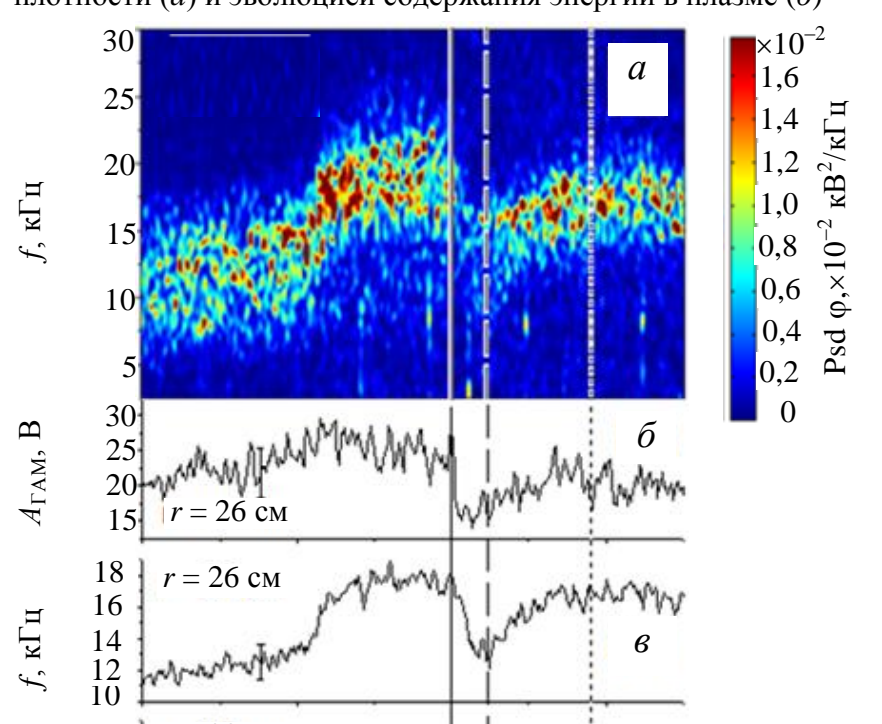

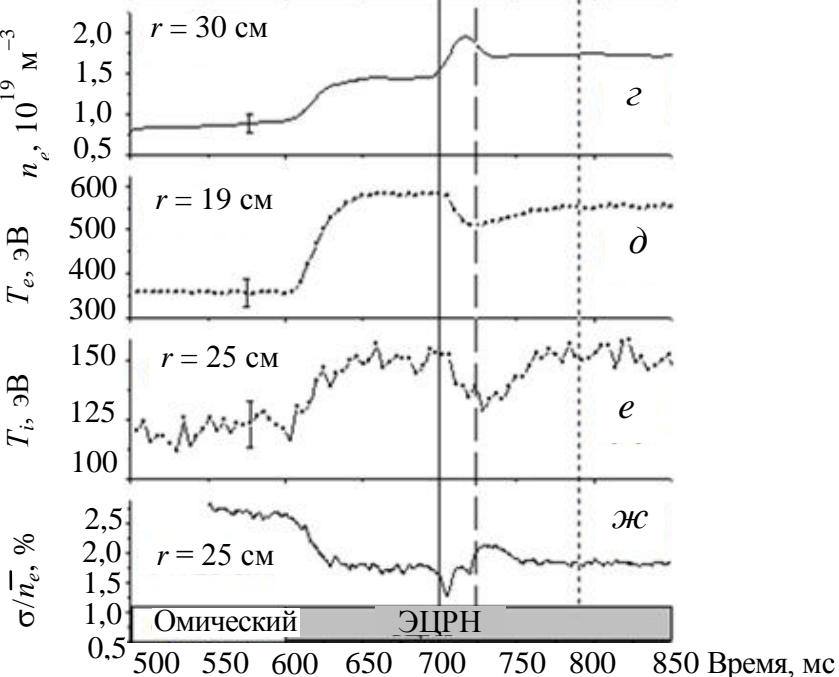

Рис. 7. Эволюция ГАМ в импульсе № 65489 с напуском Не: $a$ - спектрограмма плотности мощности колебаний потенциала; б - амплитуда ГАМ; в - частота ГАМ; гкраевая плотность; $\partial-$ электронная температура; $e-$ ионная температура (полученная по набору импульсов (№ $65488-$ 65 492) из одного режима); ж - уровень турбулентности (импульсы из одного режима); —— - напуск гелия; - - минимум частоты; - - - примерно на старте нового стационара диагностики на аппроксимационный профиль [14] с учётом шафрановского сдвига, который рассчитывается по известным параметрам разряда (ток плазмы, магнитное поле, напряжение на обходе и эффективный заряд).

Полученные калибровочные коэффициенты затем использовались при построении профиля электронной температуры во время ЭЦР-нагрева. Такой подход к измерениям позволил избежать ошибок, связанных с возможным изменением чувствительности ЕСЕ за время проведения серии импульсов. Абсолютные значения температуры были получены из показаний полупроводникового рентгеновского детектора, проводящего измерения вдоль центральной хорды.

Электронная среднехордовая (центральная хорда) плотность получена с помощью интерферометрической диагностики, эволюция уровня флуктуаций электронной плотности получена с помощью корреляционной рефлектометрии. Эволюция плотности и её профили получены абелизацией данных интерферометрической диагностики по 13 хордам интерферометра.

В исследованном режиме с напуском примесей ширина пика ГАМ больше, чем в примере на рис. 4 . Кроме того, в этом импульсе отсутствует сателлит.

Влияние импульсного напуска примеси на средний уровень потенциала (на примере импульса № 65 489). Средний уровень потенциала (усреднённое значение, исключающее вклад флуктуаций и шума аппаратуры), имеющего в данном разряде отрицательные значения, после импульса газонапуска увеличивается по абсолютной величине (рис. 6). Измерения проводились на малом радиусе $r=26$ см. Это изменение носит достаточно кратковременный характер ( 25-30 мс, порядка времени удержания в этом импульсе), однако длится значительно дольше времени напуска гелия ( $\sim 3$ мс). Затем потенциал начинает восстанавливаться, абсолютное значение его среднего уровня падает за 30-35 мс. После этого среднее значение потенциала достигает некоторого нового уровня. Эволюция потенциала при изменении плотности для квазистационарных фаз разряда с учётом погрешности согласуется с результатами предыдущих исследований [3].

При сравнении эволюции потенциала с ходом энергосодержания и плотности (см. рис. 6) можно отметить, что экстремум потенциала несколько запаздывает от минимума энергосодержания.

Влияние импульсного напуска примеси на ГАМ. СПМ показана на рис. 7, $a$. Видно, что амплитуда ГАМ резко падает и затем медленно вос- 
станавливается. Частота ГАМ также падает. Частота и амплитуда ГАМ показаны на рис. 7, 6, , в. Как упоминалось, мгновенные значения частоты и амплитуды ГАМ распределены вокруг своих средних значений. Однако изменение частоты и амплитуды, вызванное напуском примеси, превышает стандартное отклонение и может быть чётко разрешено. Также заметим, что амплитуда и частота ГАМ имеют меньшие значения на новом стационаре. Другие плазменные параметры также резко изменяются в момент напуска. На рис. 7, $\partial, e$ представлены электронная и ионная температуры. Эволюция ГАМ после напуска гелия имеет ту же форму и временной масштаб, как и эволюция температуры. Затем амплитуда и частота ГАМ выходят на новый стационарный уровень.

Влияние типа примеси на эволюцию ГАМ. Были проведены эксперименты с напуском других примесей в омических и ЭЦРН-режимах. Полученные спектрограммы показаны на рис. 8. На рис. 8, $a$ показан пример с напуском азота на фоне дополнительного ЭЦР-нагрева, на рис. $8, \sigma-$ пример с напуском гелия при омическом нагреве, на рис. 8, внапуск неона при омическом нагреве. Во всех случаях и амплитуда, и частота ГАМ уменьшаются после импульса напуска примеси. Таким образом, во всех исследованных случаях наблюдается эффект, схожий с тем, который наблюдается при напуске гелия в режиме с ЭЦРН. При этом наблюдаются некоторые отличия в деталях: ГАМ может пропадать до уровня шума на период времени около 30 мс, в то время как в некоторых импульсах она не пропадает полностью сразу после напуска (как в рассмотренном ранее импульсе № 65 489).

Сравнение эволюции частоты с теоретической. В рассмотренном импульсе № 65489 с помощью магнитных зондов и НIBР наблюдается низкочастотная МГД-активность примерно на частоте 610 кГц. Её частотный диапазон частично перекрывается с частотным диапазоном ГАМ. Для разделения ГАМ и МГД-колебаний мы приняли во внимание спектрограмму колебаний магнитного поля. С помощью НIBР можно измерить его относительные колебания $[15,16]$. Флуктуации магнитного поля пропорциональны смещению зондирующего пучка в тороидальном направлении $Z d$. Спектры колебаний электрического потенциала и $Z d$ показаны на рис. $9, a$, также показаны данные одного из магнитных зондов (рис. 9, б).

Чтобы сравнить экспериментально измеренную частоту ГАМ с теоретической $[17,18] f_{\mathrm{GAM}}=1 / 2 \pi R$ $\sqrt{\left(\left(T_{e}+7 / 4 T_{i}\right) / m_{i}\right)}$ (здесь $T_{e}$ - электронная температура, $T_{i}$ - ионная температура, $m_{i}$ - ионная масса, $R$
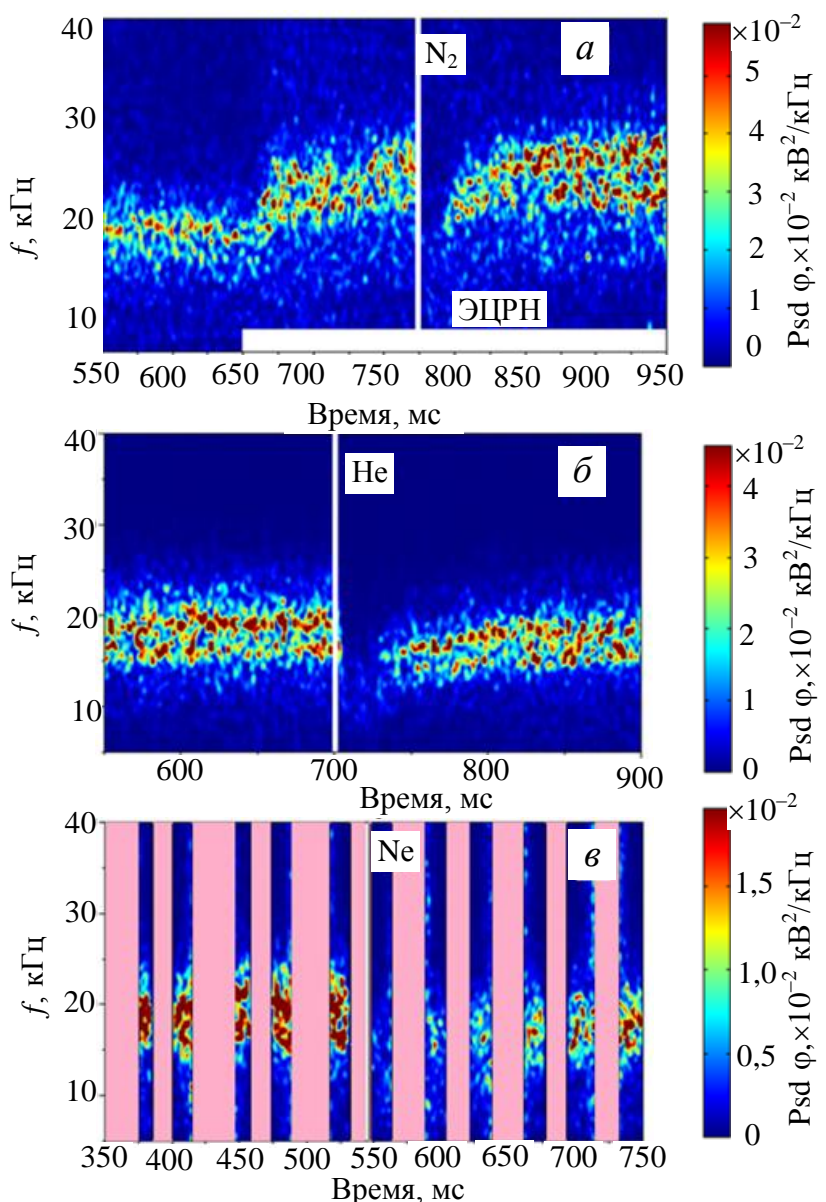

Рис. 8. Спектрограммы плотности мощности колебаний потенциала: $a$ - ЭЦР-нагрев, напуск азота (около 773 мс), радиус измерений около 21 см (\#66515); б - омический нагрев, напуск гелия (700 мс), радиус измерений -23 см (\#64883); в - омический нагрев, напуск неона (около 544 мс), данные диагностики НIBP в окрестности 23-24 см (\#65453, режим сканирования диагностики, розовым цветом обозначены области, в которых данных нет)
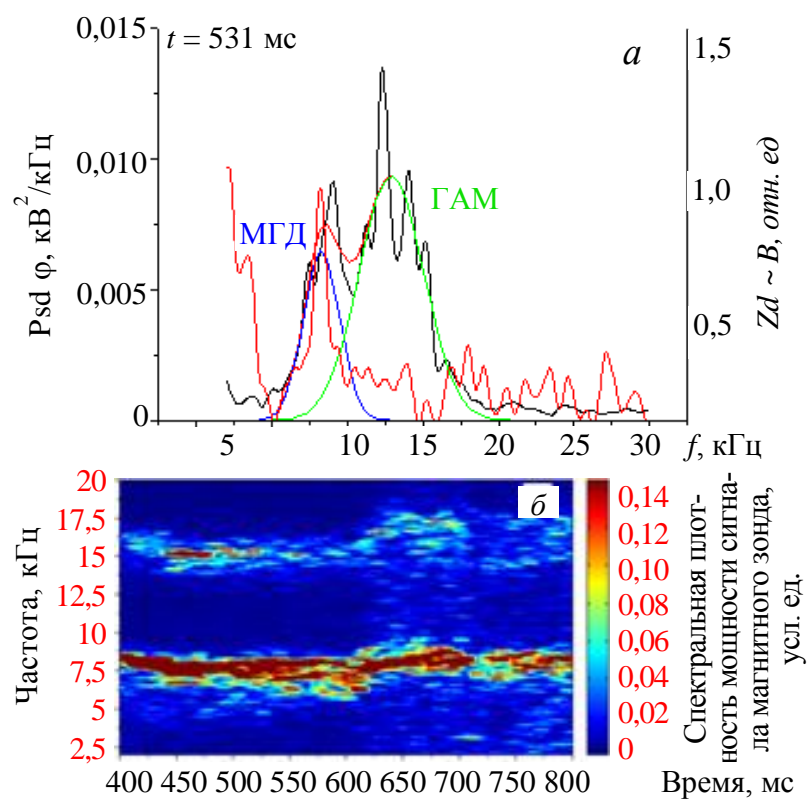

Рис. 9. Спектры колебаний электрического потенциала и сигнала НIBP диагностики $Z d \sim B(a)$; спектрограмма колебаний магнитного поля по данным магнитного зонда, расположенного в экваториальной плоскости со стороны слабого магнитного поля (б) 


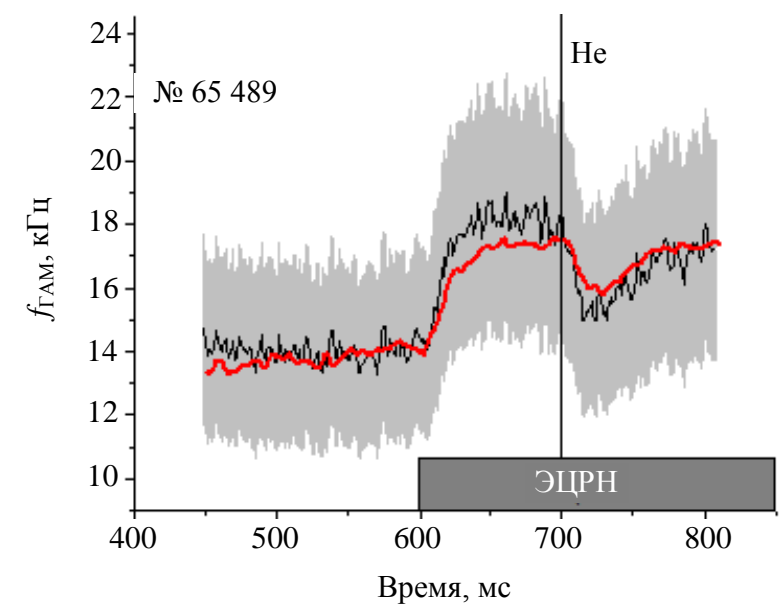

Рис. 10. Сравнение теоретической (красная линия) и экспериментальной (чёрная линия) временных эволюций частоты ГАМ большой радиус токамака), мы оценивали электронную температуру на периферии $T_{e}(\rho \sim 0,85)$, которую в данном эксперименте нельзя было корректно измерить, следующим образом. Использовалось значение $T_{e}(\rho \sim 0,64)$ в фазе ЭЦР-нагрева, нормированное на его значение в омической фазе разряда, в предположении малого изменения профиля температуры (использованный алгоритм устойчив как к очень пикированным профилям температуры с большими шафрановскими смещениями, так и к уплощённым в центре). На рис. 10 показано сравнение теоретической и экспериментальной временных эволюций частоты ГАМ (нижняя частота ГАМ принята равной 10 кГц, предполагая, что ниже 10 кГц ГАМ очень сильно зашумлена МГД-активностью).

\section{ОБСУЖДЕНИЕ РЕЗУЛЬТАТОВ}

Средний уровень потенциала связан с удержанием плазмы: чем лучше удержание, тем он более отрицательный $[2,3]$. Это справедливо для омических режимов, а также для режимов с нагревом с помощью ЭЦР и нейтральной инжекции, что было подтверждено в экспериментах на нескольких установках $[19,20]$. Величина потенциала плазмы становится более отрицательной после напуска гелия за время того же масштаба, за которое меняется частота ГАМ. Резкое изменение в момент напуска может свидетельствовать о перестройке профилей. Вопрос о том, как именно в таком процессе потенциал связан с удержанием, остаётся открытым. После нестационарной фазы потенциал выходит на новый средний уровень.

Так как частота ГАМ пропорциональна корню второй степени из $T_{e}+7 / 4 T_{i}$, то её уменьшение может быть частично объяснено уменьшением температуры на краю плазмы. На рис. 7, 2 показана локальная (краевая) плотность. Её изменения имеют тот же временной масштаб. Таким же образом изменения в плотности могут влиять на амплитуду ГАМ [9]. Однако резкое и сильное падение амплитуды ГАМ сразу после напуска примеси нельзя объяснить одним только повышением плотности. Кроме того, плотность нарастает достаточно медленно по сравнению с характерным временем падения амплитуды моды.

На рис. 7, ж показана эволюция уровня турбулентности, измеренная с помощью корреляционной рефлектометрии. Так как ГАМ и турбулентность связаны, то изменение турбулентности может влиять на амплитуду ГАМ. Сравнение с теоретическими предсказаниями показано на рис. 10. В нестационарной фазе разряда сразу после напуска примеси сильно меняются профили параметров во всем плазменном шнуре, поэтому к оценке следует подходить с осторожностью. Дополнительные сложности в анализ вносит тот факт, что нельзя точно определить частоту ГАМ при резком и сильном падении её амплитуды.

Тип примеси $\left(\mathrm{He}, \mathrm{N}_{2}\right.$ или $\left.\mathrm{Ne}\right)$ и метод нагрева не влияют на наиболее характерные особенности влияния примесей на ГАМ (уменьшение частоты и амплитуды). ГАМ в условиях многоионного состава плазмы рассматривались в [21, 22], а также влияние наличия примесей рассматривалось в [23, 24]. Частота ГАМ оказывается несколько ниже в импульсах с наличием примесей по сравнению с чистыми импульсами. Это согласуется с полученными в этой работе данными для стационарных фаз разряда.

\section{ВЫВОДЫ}

Напуск примесей сильно влияет на средний уровень потенциала и на ГАМ. Среднее значение потенциала увеличивается по абсолютной величине, затем восстанавливается за промежуток времени около 30 мс. Экстремум потенциала немного запаздывает от минимума энергосодержания, вероятно, это связано с перестройкой профилей. Амплитуда ГАМ резко уменьшается. Частота ГАМ также уменьшает- 
ся. Затем эти параметры восстанавливаются, причём время их восстановления имеет тот же характерный масштаб, что и изменение основных параметров плазмы. Характерные особенности влияния примесей на ГАМ не зависят от метода нагрева (омический или дополнительный ЭЦР) и сорта примесей.

Работа проведена за счёт Российского научного фонда, проект 14-22-00193.

\section{REFERENCES}

1. Fujisawa A. et al. Experimental progress on zonal flow physics in toroidal plasmas. - Nucl. Fusion, 2007, vol. 47, S718-S726.

2. Melnikov A.V. et al. Study of plasma potential, its fluctuations and turbulence rotation in the T-10 tokamak. - Probl. At. Sci. Technol. Ser. Plasma Phys., 2010, vol. 6, pp. 40-42.

3. Melnikov A.V. et al. Electric potential dynamics in OH and ECRH plasmas in the T-10 tokamak. - Nucl. Fusion, 2013, vol. 53, p. 093019.

4. Winsor N., Johnson J.L., Dawson J.M. Geodesic acoustic waves in hydromagnetic systems. — Phys. Fluids, 1968, vol. 11, p. 2448.

5. Melnikov, A.V. et al. Investigation of geodesic acoustic mode oscillations in the T-10 tokamak. - Plasma Phys. Control. Fusion, 2006, vol. 48, pp. S87-S110.

6. Melnikov A.V. et al. The features of the global GAM in OH and ECRH plasmas in the T-10 tokamak. - Nucl. Fusion, 2015, vol. 55, p. 063001.

7. Dnestrovskij Y.N., Melnikov A.V., Krupnik L.I., Nedzelskij I.S. Development of heavy ion beam probe diagnostics. IEEE trans. - Plasma Sci., 1994, vol. 22, pp. 310-331.

8. Andreev V.F. et al. Experimental study of density pump-out effect with on-axis electron cyclotron resonance heating at the T-10 tokamak. - Plasma Phys. Control. Fusion, 2016, vol. 58, p. 055008.

9. Eliseev L.G., Lakhin V.P., Lysenko S.E., Melnikov A.V., Zenin V.N. Dependence of the amplitude of GAMinduced core potential oscillations on density in the T-10 tokamak. - In: 43rd EPS Conf. on Plasma Physics, 2016, $\mathrm{P} 2.030$.

10. Hillesheim J.C., Peebles W.A., Carter T.A., Schmitz L., Rhodes T.L. Experimental investigation of geodesic acoustic mode spatial structure, intermittency, and interaction with turbulence in the DIII-D tokamak. - Phys. Plasmas, 2012, vol. 19.

11. Melnikov A.V. et al. Correlation properties of geodesic acoustic modes in the T-10 tokamak. - J. Phys. Conf. Ser., 2015, vol. 591, p. 012003.

12. Krupin V.A. et al. Modernized active spectroscopic diagnostics (CXRS) of the T-10 tokamak. - VANT. Ser. Termoyadernyi sintez (Problems of Atomic Science and Technology. Ser. Thermonuclear Fusion), 2014, vol. 37, issue 4, pp. 60-70 (in Russian).

13. Klyuchnikov E.A. et al. Measurements of the ion temperature at the T-10 plasma periphery based on the Doppler broadening of $5291 \AA$ spectral line of $\mathrm{C}^{5+}$ ion. - In: 43th Intern. (Zvenigorod) Conf. on Plasma Physics and Fusion Power, 2016 (in Russian).

14. Kirneva N.A. et al. Empirical formula for plasma electron temperature profile in T-10 ohmic discharges. - VANT. Ser. Termoyadernyi sintez (Problems of Atomic Science and Technology. Ser. Thermonuclear Fusion), 2014, vol. 37, issue 1, pp. 56-61 (in Russian).

15. Eliseev L.G. et al. Study of the large-scale MHD mode and its effect on GAM in the T-10 tokamak. — In: 42nd EPS Conf. on Plasma Physics, 2015, P5.159.

16. Melnikov A.V. et al. Internal measurements of Alfvén eigenmodes with heavy ion beam probing in toroidal plasmas. — Nucl. Fusion, 2010, vol. 50, p. 084023.

17. Zonca F., Chen L. Radial structures and nonlinear excitation of geodesic acoustic modes. - EPL (Europhysics Lett., 2008, vol. 83, p. 35001.

18. Smolyakov A.I., Nguyen C., Garbet X. Electromagnetic effects on geodesic acoustic and beta-induced Alfvén eigenmodes. - Nucl. Fusion, 2010, vol. 50, p. 054002.

19. Melnikov A.V., Hidalgo C., Eliseev L.G., Ascasibar E., Chmyga A.A., Dyabilin K.S., Krasilnikov I.A., Krupin V.A., Krupnik L.I., Khrebtov S.M., Komarov A.D., Kozachek A.S., López-Bruna D., Lysenko S.E., Mavrin V.A., de Pablos J.L., Pastor I., Perfilov S.V., Pedrosa M.A., Shurygin R.V., Vershkov V.A. Plasma potential and turbulence dynamics in toroidal devices (survey of T-10 and TJ-II experiments). - Nucl. Fusion, 2011, vol. 51, p. 083043. 
20. Melnikov A.V., Hidalgo C., Ido T., Shimizu A., Fujisawa A., Dyabilin K.S., Lysenko S.E. Plasma potential in toroidal devices: T-10, TJ-II, CHS and LHD. — Plasma Fusion Res., 2012, vol. 7, p. 2402114.

21. Ye L., Guo W., Xiao X., Wang S. Numerical simulation of geodesic acoustic modes in a multi-ion system. - Phys. Plasmas, 2013, vol. 20, p. 072501.

22. Sasaki M., Itoh K., Ejiri A., Takase Y. Geodesic acoustic modes in multi-Ion system. — Plasma Fusion Res., 2008, vol. 3, S1017-S1017.

23. Gurchenko A.D., Gusakov E.Z., Altukhov A.B., Selyunin E.P., Esipov L.A., Kantor M.Y., Kouprienko D.V., Lashkul S.I., Stepanov A.Y., Wagner F. Spatial structure of the geodesic acoustic mode in the FT-2 tokamak by upper hybrid resonance Doppler backscattering. — Plasma Phys. Control. Fusion, 2014, vol. 55, p. 085017.

24. Guo W., Wang S., Li J., Guo W., Wang S., Li J. Effect of impurity ions on the geodesic acoustic mode. — Physics of Plasmas, 2014, vol. 17, p. 112510.

\section{AUTHORS}

Zenin V.N. NRC “Kurchatov Institute”, pl. Akademika Kurchatova 1, 123182 Moscow, Russia; Moscow Institute of Physics and Technology, Institutskiy per. 9, 141700 Dolgoprudyi, Moscow region, Russia; vitalyzenin@mail.ru

Klyuchnikov L.A. NRC "Kurchatov Institute", pl. Akademika Kurchatova 1, 123182 Moscow, Russia; lklyuchnikov@list.ru

Melnikov A.V. NRC "Kurchatov Institute", pl. Akademika Kurchatova 1, 123182 Moscow, Russia; National Research Nuclear University MEPhI (Moscow Engineering Physics Institute), Kashirskoye shosse 31, 115409 Moscow, Russia melnikov_07@yahoo.com

Nemets A.R. NRC “Kurchatov Institute”, pl. Akademika Kurchatova 1, 123182 Moscow, Russia; Nemets_AR@nrcki.ru

Nurgaliev M.R. NRC “Kurchatov Institute”, pl. Akademika Kurchatova 1, 123182 Moscow, Russia;

Subbotin G.F. NRC “Kurchatov Institute”, pl. Akademika Kurchatova 1, 123182 Moscow, Russia; Moscow Institute of Physics and Technology, Institutskiy per. 9, 141700 Dolgoprudyi, Moscow region, Russia

Shelukhin D.A. NRC “Kurchatov Institute”, pl. Akademika Kurchatova 1, 123182 Moscow, Russia; Shelukhin_DA@nrcki.ru 